Furthermore, all major operations induce a prothrombotic diathesis. With OPCAB surgery, unlike $\mathrm{CABG}$ with $\mathrm{CPB}$, platelet numbers and function remain preserved. ${ }^{4}$ Also, a prothrombotic effect partly attributed to increased platelet reactivity has been demonstrated after OPCAB surgery. ${ }^{4}$

We have performed OPCAB surgery in 150 patients without evidence of acute coronary thrombosis. These 2 patients were the first to have OPCAB surgery with patent stents. In these patients with stented yet pristine coronary arteries, sole reliance on aspirin seems imprudent. We thus add clopidogrel. With this strategy, a further 8 patients with patent stents had uncomplicated OPCAB surgery.

\section{References}

1. Cutlip DE, Baim DS, Ho KK, Popma JJ, Lansky AJ, Cole DJ, et al. Stent thrombosis in the modern era: a pooled analysis of multicenter coronary stent clinical trials. Circulation. 2001;103:1967-71.

2. Yacoub M. Off pump coronary bypass surgery. In search of an identity. Circulation. 2001;104:1743-5.

3. Tovar EA, Borsari A. Effects of surgical manipulation on coronary stents: should surgical strategy be altered? Ann Thorac Surg. 1997;63: $37-40$.

4. Valleley MP, Bannon PG, Kritharidis L. The systemic inflammatory response syndrome and off-pump cardiac surgery. Heart Surg Forum. 2001;4(suppl I):S7-13.

\title{
Proximal prolapse of aortic intimal flap: A rare complication of acute type $A$ aortic dissection
}

\author{
G. Hossein Almassi, MD, Milwaukee, Wis
}

\section{A}

cute type A aortic dissection requires emergency surgical repair to prevent the known life-threatening complications of rupture, cardiac tamponade, acute coronary ischemia, and branched arterial occlusion. Prolapse of the intimal flap into the distal ascending aorta and the aortic arch has been reported in cases of circumferential tear of the aortic intima. ${ }^{1-4}$ We report a case of acute type A aortic dissection with intussusception of the intimal flap proximally into the aortic root and the left ventricular outflow tract, causing global myocardial ischemia.

A 75-year-old man had acute chest pain and shortness of breath. He was hypotensive, and the electrocardiogram disclosed global ST-segment depression in leads I, II, III, AVL, and AVF and precordial leads $\mathrm{V}_{2}$ to $\mathrm{V}_{6}$. Medical history included severe chronic renal insufficiency, coronary artery bypass grafting 10 years earlier, and repair of abdominal aortic aneurysm on 2 occasions 30 years and 7 years before the current presentation. A noncontrast computed tomographic (CT) scan of the chest and abdomen revealed extensive aneurysmal disease of the ascending and descending thoracic aorta with possible dissection of the descending and the remaining abdominal aorta. The patient was transferred to the cardiac catheterization laboratory, where multi-

\footnotetext{
From the Division of Cardiothoracic Surgery, Medical College of Wisconsin, Milwaukee, Wis.

Received for publication July 30, 2001; accepted for publication Feb 15, 2002.

Address for reprints: G. Hossein Almassi, MD, Division of Cardiothoracic Surgery, Medical College of Wisconsin, Milwaukee, Froedtert East Clinic, PO Box 26099, Milwaukee, WI 53226 (E-mail: galmassi@mcw.edu).

J Thorac Cardiovasc Surg 2003;125:1546-8

Copyright (C) 2003 by The American Association for Thoracic Surgery $0022-5223 / 2003 \$ 30.00+0$

doi:10.1016/S0022-5223(02)73593-9
}

ple attempts at cannulation of the coronary ostia were unsuccessful. An aortic angiogram was inconclusive for dissection of the ascending aorta and failed to show the coronary arteries. Transesophageal echocardiography revealed an intimal flap in the root of the aorta close to the aortic valve prolapsing into the left ventricle in diastole (Figure 1). The aortic valve appeared intact. The patient was transferred to the operating room in cardiogenic shock and receiving high-dose inotropic support.

Operative findings included a circumferential tear of the aortic intima in the midascending aorta at the level of old saphenous vein grafts. The intimal flap had totally prolapsed into the left ventricular outflow tract, covering the ostia of the left and right coronary arteries. During deep hypothermic circulatory arrest and retrograde cerebral perfusion, the dissection was repaired with an aortic valve conduit. Coronary artery bypass grafting was also performed.

Although infrequent, circumferential intimal tear of the ascending aorta can lead to intussusception of the intimal flap distally into the aortic arch in line with the direction of blood flow. ${ }^{1-4}$ Proximal prolapse into the aortic root and left ventricular outflow tract is very unusual. The presentation in this patient might have been due to the presence of a saphenous vein graft suture line in the ascending aorta, keeping the distal flap from prolapsing, and to an extremely low cardiac output, despite high-dose inotropic support. Aortography was not diagnostic for ascending aortic dissection because the intimal flap had intussuscepted into the left ventricular outflow tract, and therefore there was absence of a false channel in the ascending aorta. We did not find any prior report on this complication in the English literature.

Contrast-enhanced spiral CT scanning of the chest is a fast, noninvasive, and sensitive test for the diagnosis of acute aortic dissection. ${ }^{5}$ Contrast CT scan was not obtained in this patient because of severe renal insufficiency. The diagnosis was ultimately confirmed by means of transesophageal echocardiography. Transesophageal echocardiography can accurately establish the diagnosis of ascending aortic dissection by demonstrating a mobile flap or 

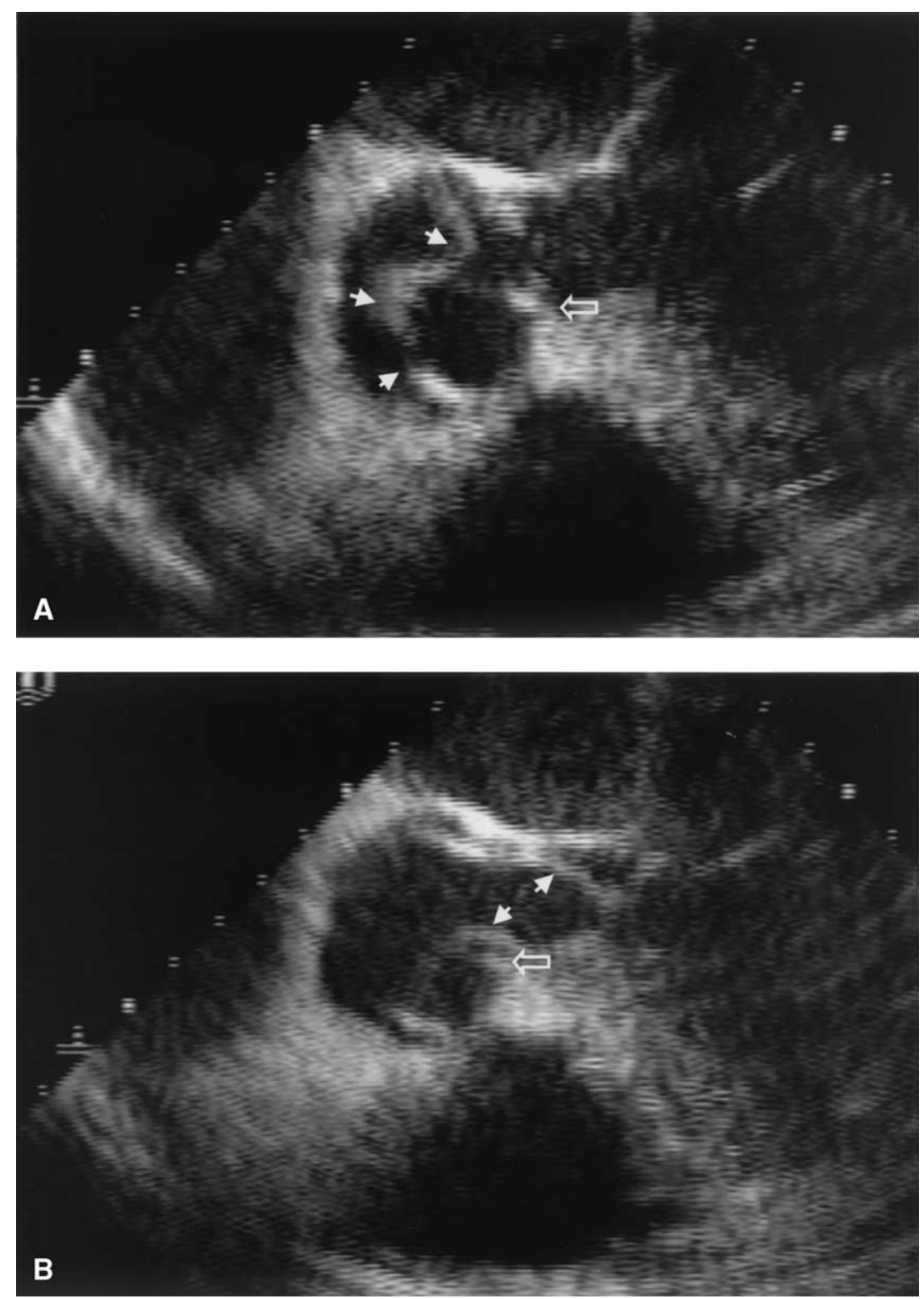

Figure 1. A, Aortic intimal flap in systole. B, Prolapsed intimal flap into the left ventricular outflow tract in diastole, covering the aortic valve. Open arrow, Aortic valve leaflet; filled arrow, intimal flap.

septum within the ascending aorta. ${ }^{5,6}$ It is, however, invasive and requires a skillful technician and physician for performance and interpretation of the findings.

Acute dissection of the ascending aorta with prolapse of the intimal flap proximally into the aortic root and left ventricular outflow tract is an extremely uncommon and potentially lethal condition. In patients with prior coronary artery bypass grafting and symptoms suggestive of aortic dissection, appropriate diagnostic procedures should be used expeditiously to establish the diagnosis in the hope of providing a timely and lifesaving surgical operation for the patient.

\section{References}

1. Svensson LC, Crawford ES. Aortic dissection. In: Svensson LG, Crawford ES, editors. Cardiovascular and vascular disease of the aorta. Philadelphia: WB Saunders; 1997. p. 42-83.

2. Reitknecht FL, Bhayana JN, Lajos TZ. Circumferential intimal tear causing obstruction of aortic arch: an unusual complication of aortic dissection. Ann Thorac Surg. 1988;46:100-1.

3. Kastan DJ, Sharma RP, Keith F, Shetty PC, Burke MW. Intimointimal intussusception: an unusual presentation of aortic dissection. AJR Am J Roentgenol. 1988;151:603-4.

4. Karabulut N, Goodman LR, Olinger GN. CT diagnosis of an unusual aortic dissection with intimointimal intussusception: the wind sock sign. J Comput Assist Tomogr. 1998;22:692-3. 
5. Nienaber CA, Kodolitsch YV, Nicolas V, Siglow V, Piepho A, Brockhoff $\mathrm{C}$, et al. The diagnosis of thoracic aortic dissection by noninvasive imaging procedures. $N$ Engl J Med. 1993;328:1-9.
6. Penco M, Paparoni S, Dagianti A, Fusilli C, Vitarelli A, Remigis FD, et al. Usefulness of transesophageal echocardiography in the assessment of aortic dissection. Am J Cardiol. 2000;86:53G-6G.

\title{
Flap suffocation: An uncommon mechanism of coronary malperfusion in acute type $A$ dissection
}

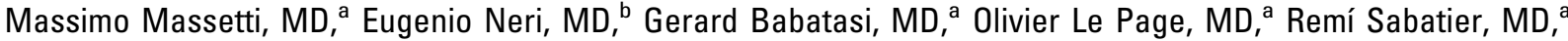 \\ Dimitrios Buklas, MD, ${ }^{a}$ Gilles Grollier, $\mathrm{MD}^{\mathrm{a}}$ and André Khayat, MD, ${ }^{\mathrm{a}}$ Caen, France, and Siena, Italy
}

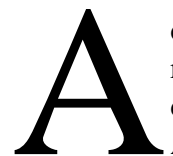

cute myocardial ischemia or infarction as a result of retrograde coronary artery dissection is a well-known complication after acute type A aortic dissection. ${ }^{1}$ Although the prevalence of chronic coronary artery disease among patients with acute dissection of the ascending aorta has been reported to be between $8 \%$ and $41 \%,{ }^{2}$ retrograde dissection of the aortic root reaching the coronary ostia is the most typical cause of acute coronary malperfusion observed in this condition. ${ }^{1}$ However, other mechanisms of myocardial ischemia, related to the diastolic expansion of the false aortic channel, have been described by Borst. $^{3}$

We present here the case of a patient with acute type A aortic dissection in whom an acute coronary malperfusion was sustained by an atypical mechanism of flow obstruction.

\section{Clinical Summary}

A 49-year-old man with a history of hypertension and sarcoidosis was transferred from an outlying hospital to our institution in severe cardiogenic shock. Two hours previously, he had experienced the sudden onset of acute chest pain, followed within a few minutes by shortness of breath and severe hypotension. A 12-lead electrocardiogram showed diffuse ST-segment depression and Twave inversion with an anterior ST elevation (without a related Q wave). A transthoracic echocardiogram was not of diagnostic quality because of a poor acoustic window. Intravenous heparin, inotropic support, and mechanical ventilation had been given without any change in the patient's condition. Systemic fibrinolysis was administered without improvement. At the time of arrival at

From the Thoracic and Cardiovascular Surgery Department, University Hospital of Caen, France, ${ }^{a}$ and the Vascular Surgery Department, University Hospital of Siena, Italy. ${ }^{\text {}}$

Received for publication Aug 10, 2002; accepted for publication Sept 9, 2002.

Address for reprints: Massimo Massetti, MD, Thoracic and Cardiovascular Surgery Department, University Hospital, 14033, Caen, France (E-mail: massetti-m@chu-caen.fr).

J Thorac Cardiovasc Surg 2003;125:1548-50

Copyright $\odot 2003$ by The American Association for Thoracic Surgery $0022-5223 / 2003 \$ 30.00+0$

doi:10.1016/S0022-5223(03)00047-3 our institution, the patient had a blood pressure of $68 / 45 \mathrm{~mm} \mathrm{Hg}$ and an irregular pulse of 110 beats/min. The peripheral pulses were weak but symmetric. Preoperative creatine kinase MB levels were not significantly elevated.

Transesophageal echocardiography was performed to obtain an analysis of the left ventricular segmental wall motion before urgent coronary angiography; surprisingly, it demonstrated the presence of an intimal flap in the ascending aorta with a circumferential distal disruption of the inner aortic wall layers. The cylindric flap presented a proximal invagination movement through the aortic valve, inside the left ventricle, during diastole (Figure 1). This diastolic intussusception of the tubular flap determined the flow obstruction of the coronary ostia and the subsequent myocardial malperfusion. Global biventricular wall motion abnormalities and a left ventricular ejection fraction of 0.25 were demonstrated. We decided to operate on the patient without delay.

The chest was opened through a median sternotomy, and the pericardium was entered. Minimal bloody pericardial effusion was detected. Severe biventricular distention and global hypokinesia were present. The dilated ascending aorta consisted of only a thin, bluish layer of tissue; the dissection terminated $2 \mathrm{~cm}$ proximal to the origin of the brachiocephalic trunk. Mildly hypothermic $\left(32^{\circ} \mathrm{C}\right)$ cardiopulmonary bypass was instituted between the right atrium and right axillary artery; the ascending aorta was crossclamped in a nondissected zone, and retrograde blood cardioplegia was administered.

On opening the aorta, we found a circumferential tear of the intima that had caused the described proximal intussusception. Above the level of the right coronary ostium, the tubular flap was partially lacerated, making residual coronary arterial perfusion still possible. The aorta below the sinotubular junction was spared. Surgical treatment consisted of the complete resection of the dissected portion of the ascending aorta and, after generous mobilization of the aortic arch and of the heart (by freeing the inferior vena cava at its diaphragmatic passage), a direct aortoaortic anastomosis with a 4-0 polypropylene suture. Despite the short duration of the aortic repair, the use of controlled reperfusion, and the absence of bleeding complications (although the patient had received systemic fibrinolysis), weaning from cardiopulmonary bypass was complex as a result of poor left ventricular performance. Cardiac compression by sternal closure was not tolerated; delayed sternotomy closure was therefore necessary. Inotropic requirements decreased from the immediate postoperative period to the 